\section{Study of intra- abdominal pressure elevation after mastectomy in dogs}

\section{Styliani Kleftouri ${ }^{1}$, Maria Vafeiadou², George Kazakos ${ }^{3}$, Erika Kirmanidou ${ }^{3}$, Lysimachos Papazoglou ${ }^{3}$}

\author{
1 Private Practice, Thessaloniki, Greece \\ 2 Private Practice, Limassol, Cyprus \\ 3 Aristotle University of Thessaloniki, Thessaloniki, Greece
}

\section{OBJECTIVES}

To assess the intra-abdominal pressure (IAP) after regional mastectomy as well as to evaluate the predisposing factors and the clinical significance of its potential increase.

\section{METHODS}

Nineteen female dogs that underwent regional mastectomy were enrolled in a prospective study. IAP was measured five times; preoperatively, at the end of surgical procedure and 1, 6 and 18 hours postoperatively. Simultaneously, the arterial pressure as well as the urine output were monitored, abdominal perfusion pressure (APP) was calculated and a pain score was determined.

\section{RESULTS}

Preoperatively, mean IAP was $4.56 \mathrm{~mm} \mathrm{Hg}$, with $1.40 \mathrm{~mm}$ $\mathrm{Hg}$ standard deviation (SD). These figures were elevated in the postoperative measurements, with the maximum being detected 1 hour after surgery at $8.58 \mathrm{~mm} \mathrm{Hg}$ mean IAP (SD: 2,9 $\mathrm{mm} \mathrm{Hg}$ ), remaining at similar levels for the rest study period. The mean arterial pressure was slightly increased postoperatively, while the urine output did not fall below $1 \mathrm{ml} / \mathrm{kg} / \mathrm{h}$. Furthermore, APP was kept above $70 \mathrm{mmHg}$ throughout the whole study. Although pain was at low levels, in 4 cases with a greater score than the average, IAP appeared higher in all postoperative measurements. Moreover, IAP showed a tendency to increase the more extended the mastectomy was.

\section{STATEMENT (CONCLUSIONS)}

An elevation of the intra-abdominal pressure can be observed postoperatively in dogs undergoing regional mastectomy, presumed due to the tension caused in the abdominal wall after the closure of the skin. This indicates that these pressure alterations should be identified and controlled for possible clinical impacts.

\section{Gastrostomy feeding tube placement in dogs with septic peritonitis: a retrospective study of 54 dogs}

\section{Kine Elmenhorst ${ }^{1}$, Pablo Perez Lopez ${ }^{2}$, Alex Belch ${ }^{3}$, Jackie L. Demetriou}

1 PDSA Croydon, London, United Kingdom

2 Dick White Referrals, Six Mile Bottom, United Kingdom

3 Langford Vets Small Animal Referral Hospital, Bristol,

United Kingdom

\section{OBJECTIVES}

To describe the utility and determine the safety of surgically placed gastrostomy feeding tubes in dogs with septic peritonitis.

\section{METHODS}

A retrospective analysis of fifty-four dogs from one institution with septic peritonitis that had undergone surgical exploration that either had or did not have a gastrostomy tube (De Pezzer or Foley) placement as part of the surgical procedure. Postoperative recovery time, hospitalisation time, complication rate and overall survival time were documented

\section{RESULTS}

Eleven dogs had no gastrostomy tube placed, fifteen dogs had a Foley gastrostomy tube placed and twenty-three dogs had a de Pezzer gastrostomy tube placed. The most common cause of septic peritonitis was dehiscence of an enterotomy or enterectomy site. The median time from surgery to first intake of enteral nutrition was 18 hours (range 3-60 hours). No major complications related to the gastrostomy tube were seen, and minor complications were seen in $32 \%$ and $13 \%$ of the de Pezzer and the Foley gastrostomy group respectively. The overall median time spent in hospital were 5 days (range 3-29 days) in patients surviving to discharge, and an overall survival rate of $52 \%$ were recorded. There were no differences between the three groups in terms of time spent in hospital or survival rate.

\section{STATEMENT (CONCLUSIONS)}

This study suggests that gastrostomy feeding tubes are a straightforward method of providing enteral nutrition to dogs with septic peritonitis, have similar complication rates to placement in non-septic patients and do not appear to cause any detrimental effect on survival rate. 